\title{
Un projet du Centre International Blaise Pascal : l'édition électronique des Pensées
}

Dominique Descotes et Gilles Proust

\section{(2) OpenEdition}

12 Journals

Édition électronique

URL : http://journals.openedition.org/ccibp/486

DOI : $10.4000 /$ ccibp.486

ISSN : 2493-7460

Éditeur

Centre international Blaise Pascal

\section{Édition imprimée}

Date de publication : 15 décembre 2008

Pagination : 2-14

ISBN : 978-2-84516-406-2

ISSN : 0249-6674

\section{Référence électronique}

Dominique Descotes et Gilles Proust, « Un projet du Centre International Blaise Pascal : l'édition électronique des Pensées », Courrier du Centre international Blaise Pascal [En ligne], 30 | 2008, mis en ligne le 30 novembre 2015, consulté le 14 septembre 2020. URL : http://journals.openedition.org/ ccibp/486

Ce document a été généré automatiquement le 14 septembre 2020.

Centre international Blaise Pascal 


\title{
Un projet du Centre International Blaise Pascal : l'édition électronique des Pensées
}

\author{
Dominique Descotes et Gilles Proust
}

\section{I/ Présentation du projet}

1 Le projet d'une édition électronique des Pensées de Pascal remonte aujourd'hui à près de six ans. La mode est actuellement aux éditions électroniques commerciales, destinées soit à offrir des photographies d'exemplaires inaccessibles à la vente, soit à fournir des textes saisis en vue de permettre des recherches lexicales aisées par ordinateur. Le défaut de telles entreprises et évidemment de se réduire à des reproductions ou des retranscriptions dépourvues de toute réflexion sur la nature particulière des textes. Cela n'a en soi rien de surprenant: entamer une réflexion analytique de fond qui permette de créer un outil informatique particulier exige de très longs délais, un engagement financier et humain lourd, et surtout une connaissance critique approfondie des textes. Or nous avons la chance à Clermont de pouvoir faire coopérer un chercheur littéraire et un analyste informatique.

Une édition électronique des Pensées est pour ainsi dire d'utilité publique. En premier lieu en raison de la nature particulière de l'ouvrage, texte majeur de la littérature française. En second lieu, parce que toutes les recherches sérieuses sur les Pensées passent aujourd'hui par l'analyse du manuscrit original et des Copies, ainsi que des premières éditions originales de Port-Royal et de leurs épigones. Or l'original des Pensées n'étant accessible que dans des conditions très difficiles, en raison de son caractère unique, et de sa valeur intrinsèque, il est encore nécessaire de recourir à des substituts, notamment l'édition phototypique de Brunschvicg ou la reproduction du manuscrit par L. Lafuma, les deux étant imprimées en noir et blanc et de façon incomplète. Quant aux Copies, dont la nécessité est peut-être moins visible au premier abord, on s'est aperçu, depuis les éditions procurées par Louis Lafuma et Philippe 
Sellier, que leur connaissance est aussi indispensable que celle de l'original. La nécessité d'une édition électronique s'est du reste fait assez vite sentir, et les éditions Champion ont répondu à ce type de besoin en publiant un $\mathrm{CD}$ rom consacré à l'ensemble de l'œuvre littéraire de Pascal, à l'exception des traités mathématiques et physique.

3 Notre objectif est différent. Plus restreint d'une certaine manière, dans la mesure où il ne concerne que les Pensées, à l'exclusion des autres textes. Plus ample dans la mesure où il implique une réflexion spécifique, en vue de créer un instrument parfaitement adapté à la nature du texte, et aux différentes formes qu'il a pu revêtir.

4 Nous bénéficions d'une certaine expérience dans ce domaine. Un premier travail a abouti en 1999 à la réalisation du CD rom multimédia sur les Lettres de A. Dettonville, à partir de l'exemplaire des traités de Pascal sur la roulette déposés à la Bibliothèque Communautaire et Universitaire de Clermont-Ferrand. Cette expérience permettait de donner un aperçu d'un document imprimé ancien, en évitant provisoirement les difficultés particulières qu'aurait posé la reproduction d'un manuscrit. En revanche, la publication en CD-rom des actes du colloque Le droit à ses époques: de Pascal à Domat en 2003 a permis d'aborder la présentation de documents iconographiques et d'expérimenter des modes de présentation de manuscrits ne posant pas des problèmes aussi complexes que l'original des Pensées. Ces essais ont fait l'objet d'une réflexion sur les meilleures solutions à choisir pour adapter les moyens informatiques nécessaire à la reproduction des textes des Pensées.

5 Il fallait d'abord effectuer un travail de fond de connaissance et d'exploration des Pensées. À cet effet, nous pouvions prendre appui sur un réseau de correspondants, spécialistes et amis de Pascal. La réflexion sur les textes a trouvé un lieu naturel dans le séminaire organisé par Laurent Thirouin, Antony McKenna et Dominique Descotes, qui procède depuis près de six ans à l'étude des fragments, tant du point de vue de leur signification que de la matière concrète des papiers, des manuscrits, et dans les métamorphoses des éditions successives. La publication des comptes rendus sur le réseau Internet ${ }^{1}$ permet d'étendre l'enquête à l'échelle mondiale. L'intérêt de ce travail collectif consiste surtout à recenser les problèmes que posent les manuscrits et les éditions, soit pour le sens, soit pour l'établissement des textes, soit pour les métamorphoses qu'il a subies. Plus utile encore était la possibilité offerte par le séminaire de recenser les besoins des chercheurs, la nature de leurs exigences en matière d'édition et d'interprétation, les difficultés qu'ils peuvent rencontrer dans leur travail, et la nature des documents dont ils ont besoin pour résoudre les problèmes qu'ils rencontrent. De ce point de vue, il a été indispensable de ne jamais séparer la recherche de la technique qui en sera le support. Gilles Proust, ingénieur informaticien au CERHAC, a très tôt pris l'habitude d'assister activement aux séances du séminaire, ce qui l'a mis en mesure de recenser les exigences d'une édition qui vise à être un instrument de recherche.

6 Le but de cette édition n'est pas en effet de fournir une matière brute, analogue à la reproduction du texte en format pdf, ni même de fournir un instrument de recherche lexicale sur le texte des Pensées. Il s'agit bien de proposer un outil permettant de procéder à des recherches fines, d'effectuer des comparaisons, et de faire des explorations et des découvertes sur ce corpus quasi identique à l'original. 
7 L'expérience du CD rom sur les Lettres de A. Dettonville nous a permis de prévoir une sorte de programme documentaire attaché à chaque fragment. Seront fournis, avec la transcription figurée des fragments :

- L'édition critique du texte établie à partir de cette transcription, et les clichés des originaux ;

- L'historique de chaque fragment d'édition en édition, jusqu'aux travaux de Y. Maeda et de P. Ernst ;

- La bibliographie des éditions et des études ;

- Les fragments et les documents connexes;

- Le relevé des sources connues, et les textes dont le rapprochement peut-être utile à l'interprétation;

- Les références et le résumé des commentaires les plus importants ;

- L'analyse de fond de texte.

Nous avons pris en compte tous les manuscrits originaux et les imprimés disponibles : le manuscrit original, dont nous disposons d'une reproduction pour la première fois en couleurs, d'une qualité exceptionnelle, et dont les folios ne sont pas rognés aux bords, comme c'est le cas dans l'édition phototypique de Brunschvicg, ce qui fait apparaître nombre d'informations souvent indispensables ; les deux Copies, elles aussi en couleurs, ce qui permet de reconstituer l'histoire des corrections que les éditeurs de Port-Royal ont apportées au texte original; le manuscrit de Sainte-Beuve, et plusieurs autres documents du même ordre. La campagne de microfilmage des originaux que le CIBP a menée dans les différentes bibliothèques de France depuis plusieurs années trouve ici son aboutissement. Parmi les éditions imprimées, sont collationnées toutes les originales, à commencer par les différents états de la pré-originale de 1669, les états successifs de l'originale de 1670, et l'édition de 1678. Ont été également prises en compte toutes les éditions subséquentes qui ont fait date dans l'histoire des Pensées et de la critique pascalienne : les textes publiés par Desmolets, et Colbert de Croissy, les éditions de Condorcet, Bossut et Renouard, le rapport de Victor Cousin, les éditions Faugère, Havet et Brunschvicg, l'édition paléographique de Tourneur, celles de L. Lafuma, de P. Sellier et de M. Le Guern, sans omettre l'analyse des documents annexes par Jean Mesnard, évidemment.

9 Les concordances entre ces éditions et manuscrits n'ont pas été établies de manière massive : les textes ont été segmentés, de manière à permettre de déterminer non pas seulement à partir de quelle édition tel ou tel texte apparait, se trouve incorporé ou est au contraire supprimé dans le corpus des Pensées, mais de suivre l'évolution des différentes parties des fragments. Une histoire individuelle des fragments est ainsi possible, qui permet de mieux comprendre les réactions des lecteurs aux différentes époques.

10 On peut se faire une idée sommaire du résultat obtenu par ces recherches systématiques, grâce au tableau suivant, extrait de la concordance générale, pris sur le fragment Sel. 38, Ordre par dialogues. On y trouve :

- La situation du papier et des parties du texte dans le Recueil original et dans les Copies, puis dans les éditions de Port-Royal ;

- Les segments de texte repris dans divers manuscrits (Sainte-Beuve, Montempuys, Clémencet) et les premières éditions jusqu'au rapport de Victor Cousin ;

- La situation et l'état du texte, avec l'ordre de ses parties $(1, / 2$, etc.) dans les différentes éditions de Faugère à nos jours. 
11 Sel. 38/1 : Ordre par dialogues. «Que dois-je faire? Je ne vois partout qu'obscurité. Croirai-je que je ne suis rien? Croirai-je que je suis Dieu?»

\begin{tabular}{|l|l|l|l|}
\hline Recueil original & ${\text { Copie } C_{1}}$ & Copie C $_{2}$ & P-R 1669-1678 \\
\hline $29-1$ & $\mathrm{~N}^{\circ} 3 / 1$ p. 1 & p. 13 & Absent \\
\hline
\end{tabular}

\begin{tabular}{|l|l|l|l|l|}
\hline $\begin{array}{l}\text { Manuscrits et éditions post P-R (Colbert, } \\
\text { Desmolets) }\end{array}$ & $\begin{array}{l}\text { Éd. } \\
\text { Condorcet } \\
1776\end{array}$ & $\begin{array}{l}\text { Éd. Bossut } \\
1779 \text { tome }\end{array}$ & $\begin{array}{l}\text { Éd. } \\
\text { Renouard } \\
1803 \quad \text { tome } \\
\text { II }\end{array}$ & $\begin{array}{l}\text { Rapport } \\
\text { Cousin } \\
1842\end{array}$ \\
\hline $\begin{array}{l}\text { Ms Sainte Beuve } \\
\text { p. } 104\end{array}$ & Absent & Absent & Absent & p. 250 \\
\hline
\end{tabular}

\begin{tabular}{|l|l|l|l|l|l|}
\hline $\begin{array}{l}\text { Éd. Faugère } \\
1884\end{array}$ & $\begin{array}{l}\text { Éd. Havet } \\
1825-1866\end{array}$ & $\begin{array}{l}\text { Éd. Brunschvicg } \\
1904\end{array}$ & $\begin{array}{l}\text { Éd. Tourneur } \\
1942\end{array}$ & $\begin{array}{l}\text { Éd. Lafuma } \\
1951\end{array}$ & $\begin{array}{l}\text { Éd. Le Guern } \\
1977\end{array}$ \\
\hline t. II, p. 389/12 & $\begin{array}{l}\text { Art. XXV, } \\
n^{\circ} 109 / 1\end{array}$ & $n^{\circ} 227 / 1$ & p. 167-3/1 & $n^{\circ} 2$ & $n^{\circ} 2 / 1$ \\
\hline
\end{tabular}

Sel. 38 / 2 / « Toutes choses changent et se succèdent. » « Vous vous trompez, il y a ... »

\begin{tabular}{|l|l|l|l|}
\hline Recueil original & Copie C $_{1}$ & Copie C $_{2}$ & P-R 1669-1678 \\
\hline $29-1$ & $\mathrm{~N}^{\circ} 3 / 2$ p. 1 & p. 13 & Absent \\
\hline
\end{tabular}

\begin{tabular}{|l|l|l|l|l|}
\hline $\begin{array}{l}\text { Manuscrits et éditions post P-R (Colbert, } \\
\text { Desmolets) }\end{array}$ & $\begin{array}{l}\text { Ed. } \\
\text { Condorcet } \\
1776\end{array}$ & $\begin{array}{l}\text { Ed. Bossut } \\
1779 \text { tome } \\
\text { II }\end{array}$ & $\begin{array}{l}\text { Ed. } \\
\text { Renouard } \\
1803 \text { tome II }\end{array}$ & $\begin{array}{l}\text { Rapport } \\
\text { Cousin } \\
1842\end{array}$ \\
\hline $\begin{array}{l}\text { Ms Sainte Beuve } \\
\text { p. } 104\end{array}$ & Absent & Absent & Absent & p. 250 \\
\hline
\end{tabular}

\begin{tabular}{|l|l|l|l|l|l|}
\hline $\begin{array}{l}\text { Éd. Faugère } \\
1884\end{array}$ & $\begin{array}{l}\text { Éd. Havet } \\
1825-1866\end{array}$ & $\begin{array}{l}\text { Éd. Brunschvicg } \\
1904\end{array}$ & $\begin{array}{l}\text { Éd. Tourneur } \\
1942\end{array}$ & $\begin{array}{l}\text { Éd. Lafuma } \\
1951\end{array}$ & $\begin{array}{l}\text { Éd. Le Guern } \\
1977\end{array}$ \\
\hline t. II, p. 389/13 & $\begin{array}{l}\text { Art. XXV, } \\
n^{\circ} 109 / 2\end{array}$ & $n^{\circ} 227 / 2$ & p. $167-3 / 2$ & $n^{\circ} 3 / 1$ & $n^{\circ} 2 / 2$ \\
\hline
\end{tabular}

Sel. 38 / 3 : «Et quoi ne dîtes-vous pas vous-même que le ciel et les oiseaux prouvent Dieu? » [...] néanmoins cela est faux à l'égard de la plupart. 


\begin{tabular}{|l|l|l|l|}
\hline Recueil original & Copie $_{1}$ & Copie $_{2}$ & P-R 1669-1678 \\
\hline $29-1$ & $\mathrm{~N}^{\circ} 4$ p. 1 & p. 13 & Absent \\
\hline
\end{tabular}

\begin{tabular}{|l|l|l|l|}
\hline Manuscrits et éditions post P-R (Colbert, Desmolets) & $\begin{array}{l}\text { Éd. Condorcet } \\
1776\end{array}$ & $\begin{array}{l}\text { Éd. Bossut } \\
1779 \text { tome II }\end{array}$ & Rapport Cousin \\
\hline Ms Sainte Beuve p. 104 & Absent & Absent & p. 250 \\
\hline
\end{tabular}

\begin{tabular}{|c|c|c|c|c|c|}
\hline $\begin{array}{l}\text { Éd. Faugère } \\
1884\end{array}$ & $\begin{array}{l}\text { Éd. Havet } \\
\text { 1825-1866 }\end{array}$ & $\begin{array}{l}\text { Éd. Brunschvicg } \\
1904\end{array}$ & $\begin{array}{l}\text { Éd. Tourneur } \\
1942\end{array}$ & $\begin{array}{l}\text { Éd. Lafuma } \\
1951\end{array}$ & $\begin{array}{l}\text { Éd. Le Guern } \\
1977\end{array}$ \\
\hline t. II, p. $389 / 14$ & $\begin{array}{l}\text { Art. XXV, } \\
n^{\circ} 200\end{array}$ & $n^{\circ} 244$ & p. $167-3 / 3$ & $n^{\circ} 3 / 2$ & $n^{\circ} 2 / 3$ \\
\hline
\end{tabular}

Sel. 38 / 4 : Lettre pour porter à rechercher Dieu. Et puis le faire chercher chez les philosophes, pyrrhoniciens et dogmatistes, qui travailleront celui qui les cherche.

\begin{tabular}{|l|l|l|l|}
\hline Recueil original & Copie C $_{1}$ & Copie C $_{2}$ & P-R 1669-1678 \\
\hline $29-1$ & N $^{\circ}$ p. 1 & p. 13 & Absent \\
\hline
\end{tabular}

\begin{tabular}{|l|l|l|l|}
\hline Manuscrits et éditions post P-R (Colbert, Desmolets) & $\begin{array}{l}\text { Éd. Condorcet } \\
1776\end{array}$ & $\begin{array}{l}\text { Éd. Bossut } \\
1779 \text { tome II }\end{array}$ & $\begin{array}{l}\text { Rapport Cousin } \\
1842\end{array}$ \\
\hline Absent & Absent & Absent & p. 249 \\
\hline
\end{tabular}

\begin{tabular}{|l|l|l|l|l|l|}
\hline $\begin{array}{l}\text { Éd. Faugère } \\
1884\end{array}$ & $\begin{array}{l}\text { Éd. Havet } \\
1825-1866\end{array}$ & $\begin{array}{l}\text { Éd. Brunschvicg } \\
1904\end{array}$ & $\begin{array}{l}\text { Éd. Tourneur } \\
1942\end{array}$ & $\begin{array}{l}\text { Éd. Lafuma } \\
1951\end{array}$ & $\begin{array}{l}\text { Éd. Le Guern } \\
1977\end{array}$ \\
\hline t. II, p. 390/15 & $\begin{array}{l}\text { Art. XXV, } \\
n^{\circ} 108 \text { bis }\end{array}$ & $n^{\circ} 184$ & p. 167-3/4 & $n^{\circ} 4$ & $n^{\circ} 2 / 4$ \\
\hline
\end{tabular}

L'aspect iconographique du projet offre aussi d'autres perspectives.

16 Nous proposons une présentation photographique des manuscrits, papier par papier, et des extraits correspondants des Copies, permettant d'obtenir toutes les informations disponibles, en vue d'y recueillir directement sur les originaux, outre les renseignements que nous allons fournir, ceux qu'il sentira le besoin de chercher par lui-même.

17 On peut montrer aisément comment les logiciels de manipulation d'images permettent de donner une représentation concrète de la genèse des fragments, grâce à une présentation dynamique des additions et corrections de telle manière qu'elles 
apparaissent progressivement à l'écran. On peut en saisir la procédure sur l'exemple du fragment Partis (Sel. 187 - Laf. 154 - Recueil p. 63-2.).

Le papier tel qu'il nous est parvenu dans le folio 63 du Recueil contient Sel 187 - Laf 154 et 155. textes supposés perdus. Ces découvertes prolongent celles de Salomon Reinach (1877) et de Zacharie Tourneur (1935), obtenues par les moyens disponibles à leur époque.

\section{II/ Découverte de deux textes disparus du Recueil des Pensées et hypothèse sur la disparition de deux autres textes}

Folios [226] et [227]

27 [Image non convertie]

Cliché BNF

Etat 3 : Sel. 187 - Laf 154 deuxième série de corrections (en vert) (avant que ne soient barrées verticalement les lignes 2,3 et 4 )

[Image non convertie]

Cette reconstitution suppose que le mot «faux » s'applique au début de la deuxième ligne et non aux lignes 2, 3 et 4 barrées verticalement.

pourra éventuellement être paramétrée à volonté par l'utilisateur, pour lui permettre de tester ses propres hypothèses.

Une exploitation systématique des photographies originales a permis de vérifier ou d'infirmer les hypothèses de Pol Ernst, sur les reconstitutions de certains feuillets originaux du manuscrit tels qu'ils étaient avant découpage, et de proposer dans quelques cas des reconstitutions nouvelles.

Plus nouveaux encore sont certains résultats issus de l'examen minutieux des

L'une des plus curieuses porte sur les folios 226 et 227 du Recueil original.

Ces folios appartiennent au cahier 19 du Recueil. Rappelons que les folios n'étaient pas numérotés au moment du collage ; la pagination n’a été effectuée que lors de la reliure 
du Recueil, une vingtaine d'années plus tard. La personne qui est intervenue sur les cahiers $18,19,23$, et peut-être 11 , a collé les papiers sur des feuilles intermédiaires, elles-mêmes collées sur les cahiers.

Le folio 227 contient quatre papiers qui correspondent de haut en bas aux textes Laf 774-Sel 638, Laf 770-Sel 635, Laf 221-Sel 254 et Laf 222-Sel 255.

31 Sur le folio 227, on constate que la personne qui a réalisé le collage a négligé l'inscription "Voyez Perpétuité », tracée au verso du papier bas (Laf. 222) : ce dernier papier a été décollé et monté sur onglet à la demande de Zacharie Tourneur, le 6 novembre 1935, ce qui nous permet de lire cette inscription portée sur le verso (voir le cliché ci-dessus).

Le folio 226 est le verso du folio 225 ; il ne supporte aucun papier. Des traces d'encre et de colle sont cependant visibles. La feuille qui, sur le cliché, semble collée sur le folio 226 est en réalité collée sur le folio 225 , et visible en transparence du folio 226 .

On peut en l'occurrence faire deux suppositions différentes. Ou bien l'ensemble des papiers a d'abord été collé sur une feuille de support avant que celle-ci soit elle-même collée sur le Recueil, ou bien la feuille de support a d'abord été collée sur le Recueil, et les papiers ont été collés ensuite sur cette feuille. La première hypothèse semble la plus logique a priori, mais la reconstitution que nous proposons ci-dessous favoriserait plutôt la deuxième hypothèse.

On constate que quatre textes ne sont connus par les Copies $\left.C_{1}, p .401 v^{\circ}-403\right)$ et $C_{2}$, p. $375 \mathrm{v}^{\circ}-377 \mathrm{v}^{\circ}$, ne figurent pas parmi les papiers du Recueil original. Il s'agit des fragments Sel. 639 et 640 (Laf. 775), Sel. 641 (Laf. 776), et Sel. 642 (Laf. 777).

Extrait de la transcription de la Copie C1, p. $401 v^{\circ}-403$ :

\section{[Laf.774/Sel.638]}

Contre ceux qui sur la confiance

de la Misericorde de Dieu demeurent

dans la non chalance sans faire de

bonnes Cuvres.

Comme les deux sources de nos pechez sont l'Orgueil \& la paresse Dieu nous a decouvert deux qualitez en luy pour les guairir, Sa Misericorde \& Sa Jstice.

Quelque saintes que soyent les œuvres Et non intres in judicium. \&c.

Le propre de la Justice est d'abbattre l'Orgueil \& le propre de la misericorde est de combattre la paresse en invitant aux bonnes CEuvres selon ce passage, La misericorde de Dieu invite à penitence, \& cét autre des Ninivites faisons penitence pour voir si par avanture il aura pitié de nous. \& ainsy tant s'en faut que la misericorde authorise le relaschement que c'est au contraire la qualité qui le combat formellement de sorte qu'au lieu de dire [p. 403] S'il n'y avoit point en Dieu de misericorde il faudroit faire toutes sortes d'efforts pour la vertu, il faut dire au contraire que c'est parce qu'il y a en Dieu de la misericorde qu'il faut faire toutes sortes d'efforts.

\section{[Laf 775 / Sel 639 - Papier original perdu]}

Contre ceux qui abusent des passages

de l'Escriture \& qui se prevalent de ce

qu'ils en trouvent quelqu'un qui semble

favoriser leur Erreur.

Le Chapitre de vespres, le dimanche de la Passion, L'Oraison pour le Roy.

\section{[Laf 775 / Sel 640 - Papier original perdu]}

Explication de ces paroles

Qui n'est point pour moy est contre 
moy

Et de ces autres

Qui n'est point contre vous est pour

vous.

Une personne qui dit je ne suis ny pour ny contre on doit luy respondre.

39 Il est aussi de la main de Gilberte. Les parties entre crochets dans la transcription cidessous sont celles que le rognage a supprimées.

Explication de ces paroles qui n'es[t point]

Pour moy est contre moy et de ces a[utres]

Qui n'est point contre vous est pour vou[s.]

Un personne qui dit je ne suis pour ny contre ny contre on doit [luy]

repondre

[Laf 774 / Sel 638] Texte inversé

\section{[Image non convertie]}

La flèche indique la position de traces d'écriture visibles.

Cliché BNF

Agrandissement de restes du folio 226 du Recueil

[Image non convertie]

Cliché BNF 
Le texte révélé par un effet de « miroir » se présente comme ci-dessous.

Texte révélé par un effet de « miroir »

\section{[Image non convertie]}

En suppléant certaines lettres effacées ou en renforçant celles qui sont difficilement lisibles, on peut reconstituer le début de ce texte comme dessous.

Fragment transcrit dans les Copies $C_{1}$ et $C_{2}$ [Laf $776-$ Sel. 641]

\section{[Image non convertie]}

Il s'agit du fragment transcrit dans les Copies $C_{1}$ et $C_{2}$ [Laf 776 - Sel. 641] dont le papier original est perdu. En indiquant en gras les parties visibles à l'œil nu par grossissement, on rétablit :

L'histoire de l'Eglise doit proprement estre appellée l'histoire de

la verité

Ce texte est, semble-t-il aussi de la main de Gilberte.

Comment interpréter la présence de ces traces d'écriture lisibles sur les folios 226 et 227 du manuscrit original ?

Si l'on part de l'hypothèse que les Copies reflètent fidèlement l'ordre des papiers tel qu'ils étaient dans le manuscrit original, il découle que le texte perdu de Sel. 639 était nécessairement écrit sur le même papier que Sel. 638 et 640 (qui se trouve au verso de Sel. 638), et plus précisément au-dessous du fragment Sel 638 (comme le montre le schéma ci-dessous), puisque les Copies les transcrivent dans cet ordre. Si d'ailleurs Sel. 639 avait été écrit sur un autre papier ou si les deux papiers avaient été séparés par Pascal, l'ordre des Copies aurait été Sel. 638, Sel. 640 puis Sel. 639 et non 638-639-640.

\begin{tabular}{|l|l|l|l|}
\hline 226 & & $\begin{array}{l}\text { Sel 638 / Sel 640 } \\
\text { (verso) } \\
\text { Sel 639 }\end{array}$ & 227 \\
\hline & $\begin{array}{l}\text { Sel } 641 \\
\text { (décalqué } \\
\text { de bas en haut verticalement) }\end{array}$ & & \\
\hline & & & \\
\hline
\end{tabular}

Pour expliquer la disparition du texte Sel. 639 et la présence conjointe du décalque du texte Sel. 641 sur le folio 226, on peut émettre l'hypothèse que Sel. 641 se trouvait à l'origine au verso de Sel. 639. Il est fort probable que c'était aussi le cas du texte correspondant au papier perdu de Sel. 642. À l'unicité du papier semble s'ajouter celle de l'écriture: Pol Ernst supposait dans son étude sur les papiers autographes de Gilberte que les textes perdus étaient de sa main, tout aussi bien ceux qui subsistent. Ces traces semblent lui donner raison.

Reste à reconstituer le déroulement des événements qui ont abouti simultanément :

- à la perte du papier qui portait les textes Sel. 639, 641 et 642 ; 
- au fait que le papier qui portait Sel. 638 et 640 a été collé sur le folio sans qu'une fenêtre soit ménagée pour rendre visible le fragment Sel. 640 ;

- et que les traces du fragment Sel. 641 se soient trouvées imprimées sur le folio 226.

Vue schématique du « papier » au moment du collage, avant l'incident

[Image non convertie]

Il semble qu'un l'incident se soit produit au moment du collage.

L'aspect général du papier qui portait les fragments Sel. 368, 639, 640, 641 et 642 était sans doute conforme au schéma situé ci-dessous, en présentant côte à côte le recto et le verso. Nous signalons par des traits rouges les découpages effectués au moment du collage.

On peut constater que Pascal avait demandé à Gilberte de laisser un grand emplacement après le texte Sel. 640, qui se termine par («On doit lui répondre »), probablement pour le compléter.

Comme on peut le constater pour de nombreux papiers, l'auteur du collage a enlevé par découpage les parties vierges du papier, en particulier de part et d'autres des titres.

On est conduit à penser qu'il a ensuite encollé le papier sans en vérifier le verso (peutêtre en passant le papier sur un pinceau ou une spatule enduite de colle pour minimiser la quantité du produit sur le papier, opération qui ne nécessitait pas de voir le verso). Si en effet la colle avait été passée directement sur les folios et les feuilles intermédiaires, on le verrait autour des papiers. Puis l'ouvrier a sans doute commencé à coller le papier sur la feuille intermédiaire.

5 Le haut du papier étant collé sur le folio 227, il s'est aperçu - trop tard - qu'il y avait un texte au verso, en bas du papier. Il a alors coupé, entre Sel 638 et Sel 639, la partie non encore collée (voir sur la reconstitution la position des ciseaux).

Schéma de la reconstitution du collage

\section{[Image non convertie]}

À la fin de l'opération le papier coupé s'est déplacé vers la gauche sur l'autre feuille du cahier (folio 226) en faisant une rotation de $80^{\circ}$ dans le sens des aiguilles d'une montre, de sorte que le texte de Sel 641 s'est retrouvé la tête en bas légèrement incliné. Étant déjà encollé, ce texte s'est alors en partie décalqué sur ce folio. Il est probable que le texte de Sel 642 a fait de même, car il en subsiste, semble-t-il, quelques faibles traces sous le texte de Sel. 641.

Le papier découpé, sans doute trop dégradé, aura probablement été jeté.

Le papier suivant (Laf. 770 - Sel. 635) a ensuite été collé sous le papier restant en profilant de l'emplacement libéré.

Le fait que les Copies aient pris en compte les textes manquants dans l'original tend à confirmer la grande fiabilité des Copies, au moins pour ce qui touche la transcription des textes. On remarquera toutefois que, si cette reconstitution est exacte, puisque les fragments Sel. 638 à 642 en question se trouvaient à l'origine sur un seul et même papier, les éditeurs qui numérotent les textes par papier ne devraient leur affecter qu'un seul numéro. 


\section{III/ Remarques sur l'ordre des Copies}

71 L'analyse préparatoire à l'édition, appuyée sur les travaux de nos prédécesseurs, a fait apparaître des problèmes, qui ouvrent de nouveaux champs de recherche.

Les travaux de Louis Lafuma, de Philippe Sellier et de Jean Mesnard ont attiré l'attention des pascalisans sur l'importance des Copies pour l'interprétation de la compréhension du manuscrit original. On sait que l'édition Lafuma Luxembourg (1951) suit l'ordre de la première Copie, alors que Philippe Sellier (1976) a préféré suivre la seconde, qu'il considère comme copie de référence ne varietur conservée par Gilberte.

L'étude de Jean Mesnard publiée en 1971 dans Les «Pensées » de Pascal ont trois cents ans, sous le titre "Aux origines de l'édition des «Pensées »: les deux Copies», a permis de préciser les différences entre l'ordre des « unités» telles qu'elles sont placées dans chacune des deux Copies ${ }^{3}$.

Jean Mesnard repère dans ces deux Copies des « grands dossiers » consacrés à des sujets communs, qui forment en quelque sorte des unités supérieures à ce que l'on appelle d'ordinaire des liasses.

Par exemple, l'ensemble d'unités XXXII à XXXIV concerne exclusivement les miracles ; les unités XXI et XXII touchent l'esprit de géométrie et l'esprit de finesse ; l'unité XX traite le problème de la créance ${ }^{4}$.

Dans la première Copie (notée $\mathrm{C}_{1}$ ), Jean Mesnard distingue les groupements suivants.

$77 \quad 1$ à 27

(unités à titre correspondant à la « table des matières »)

Table des matières ${ }^{5}$ et I

II à XIX

$\mathrm{XX}$

XXI à XXII

XXIII à XXXI

XXXII à XXXIV

78 Dans la seconde Copie (notée $C_{2}$ ), l'ordre est différent, et, en conservant la numérotation des unités précédente, on aboutit au classement suivant, apparemment tout différent.

79 Table des matières

I

1 à 27

$\mathrm{XXXV}$

XXXII à XXXIV

XXIII à XXXI

XXI à XXII

$\mathrm{XX}$

II à XIX

$\mathrm{C}_{1}$ ne comporte pas le titre Preuves de la religion par le peuple Juif, les prophéties et quelques discours, et l'unité XXXV (Contre la fable d'Esdras) n'y figure pas non plus.

81 Il faut également noter que dans $C_{1}$, les unités sont chacune dans un cahier propre, alors que dans $C_{2}$, les unités ont été copiées continûment les unes après les autres, 
chevauchant les cahiers. De telle sorte que les unités de $C_{1}$ ont pu subir des manipulations, alors que c'était impossible dans le cas de $\mathrm{C}_{2}$.

\section{Tables des matières et I}

II à XIX

$\mathrm{XX}$

XXI à XXII

XXIII à XXXI

XXXII à XXXIV

2 Admettons que le copiste entreprenne de réaliser une copie. Il copie d'abord les unités 1 à 27 (unités à titre correspondant à la « table des matières ", puis la table des matières et I, qu'il entasse sur une première pile, dans l'ordre inverse.

Il copie ensuite les grands dossiers, qu'il entasse aussi dans l'ordre inverse, mais dans une seconde pile. 
Le copiste a au bout du compte stocké l'ensemble des grands dossiers dans l'ordre où ils seront copiés dans $\mathrm{C}_{2}$.

\begin{tabular}{|c|c|}
\hline Première pile & Deuxième pile \\
\hline $\begin{array}{l}\text { Table des matières et I } \\
1 \text { à } 27\end{array}$ & $\begin{array}{l}{[X X X V]} \\
\text { XXXII à XXXIV } \\
\text { XXIII à XXXI } \\
\text { XXI à XXII } \\
\text { XX } \\
\text { II à XIX }\end{array}$ \\
\hline
\end{tabular}

Pour des raisons qu'il serait trop long d'exprimer ici, la présence de l'unité XXXV dans $C_{2}$ et son absence dans $C_{1}$ n'infirme pas la reconstitution de ce processus.

Le lecteur pourra vérifier par lui-même que le processus est le même si l'on suppose que $\mathrm{C}_{0}$ était à l'origine dans l'ordre que reflète $\mathrm{C}_{2}$, et qu'il aboutit à transformer l'ordre de $\mathrm{C}_{2}$ en ordre de $\mathrm{C}_{1}$.

97 Les résultats ainsi obtenus permettent d'envisager d'une manière nouvelle le problème de la différence de l'ordre entre les Copies $C_{1}$ et $C_{2}$ : en réalité, cette différence est purement apparente, puisqu'une simple transformation toute naturelle permet de passer d'un ordre à l'autre, tout en conservant l'ordre des grands dossiers.

En revanche, comme ces deux opérations que nous avons décrites sont parfaitement symétriques, il n'est pas possible, dans l'état actuel de l'analyse, d'affirmer que l'une est plus probable que l'autre, et par conséquent, de dire que l'une des Copies est antérieure à l'autre.

99 Cette entreprise d'édition électronique, née et conduite par le CERHAC à ClermontFerrand, bénéficie de l'aide financière de l'Université Blaise Pascal (Clermont 2), du Centre International Blaise Pascal et des Amis du CIBP, du CNRS (UMR 5037) pour la réalisation des photos du manuscrit original, ainsi que de la région Rhône-Alpes pour celle de la Copie $\mathrm{C}_{1}$.

100 Nous devons aussi beaucoup à l'aide de Mathieu Lescuyer et Christophe Vellet, qui nous ont guidés dans nos contacts avec la BNF.

\section{NOTES}

1. http://cerhac.univ-bpclermont.fr (Rubrique Séminaire des Pensées).

2. Le texte est bien sur le folio 226 et non sur la feuille blanche collée sur le folio 225 (visible ici en transparence).

3. Mesnard Jean, " Aux origines de l'édition des "Pensées" : les deux copies", dans Les "Pensées" de Pascal ont trois cents ans, Clermont-Ferrand, De Bussac, 1971, p. 1-30. 
4. Mesnard Jean, op. cit., p. 12.

5. La table des matières et l'unité 1 sont sur le même cahier. Cette situation de la table des matières après les "liasses à titre" explique que, dans $C_{1}$, une copie en ait été placée en tête de l'ensemble.

INDEX

Mots-clés : Pascal, Pensées, édition électronique

Keywords : Pascal, Pensées, electronic publishing 